\title{
The Genome Sequence of the Emerging Common Midwife Toad Virus Identifies an Evolutionary Intermediate within Ranaviruses
}

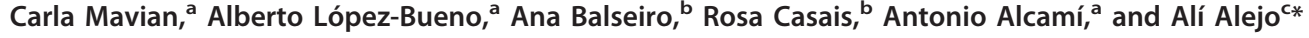 \\ Centro de Biología Molecular Severo Ochoa (CSIC-UAM), Universidad Autónoma de Madrid, Madrid, Spainª; Centro de Biotecnología Animal, Servicio Regional de \\ Investigación y Desarrollo Agroalimentario, Gijón, Spainº; and Instituto Nacional de Investigación y Tecnología Agraria y Alimentaria, Valdeolmos, Spain
}

\begin{abstract}
Worldwide amphibian population declines have been ascribed to global warming, increasing pollution levels, and other factors directly related to human activities. These factors may additionally be favoring the emergence of novel pathogens. In this report, we have determined the complete genome sequence of the emerging common midwife toad ranavirus (CMTV), which has caused fatal disease in several amphibian species across Europe. Phylogenetic and gene content analyses of the first complete genomic sequence from a ranavirus isolated in Europe show that CMTV is an amphibian-like ranavirus (ALRV). However, the CMTV genome structure is novel and represents an intermediate evolutionary stage between the two previously described ALRV groups. We find that CMTV clusters with several other ranaviruses isolated from different hosts and locations which might also be included in this novel ranavirus group. This work sheds light on the phylogenetic relationships within this complex group of emerging, disease-causing viruses.
\end{abstract}

Gisen obal population declines and extinction of multiple amphibian species have been reported over the last 20 years (11). As estimated by the International Union for Conservation of Nature, the Amphibia class includes the highest number of critically endangered species among the animal classes examined, with an estimated $41 \%$ of its species under threat (23). Therefore, this class seems to be a particularly sensitive indicator of the current biodiversity losses associated with the global warming process and other human-related environmental factors. It has been shown that habitat loss, increased human population density, and increased climatic variability are important factors that compromise the survival of amphibian species (34) and that multiple drivers of extinction are likely to coordinately accelerate amphibian declines in the near future (20).

The role of emerging infectious diseases in the rapid decline of amphibian populations is being increasingly studied. The recent spread of the lethal fungal pathogen Batrachochytrium dendrobatidis is well documented and has been associated with long-term amphibian population declines in several locations (6). A second widespread pathogen of amphibians that is now recognized as an emerging infectious disease and therefore likewise included in the list of notifiable diseases by the World Organization for Animal Health is ranaviruses. Although their association with population declines has to be studied further (14), ranavirus infections have been clearly associated with mass mortalities of several amphibian as well as reptile and fish species worldwide $(3,36,40)$.

Ranavirus is a genus within the Iridoviridae family which includes large, icosahedral viruses containing circular, doublestranded DNA genomes with sizes ranging from $105 \mathrm{kbp}$ to 140 $\mathrm{kbp}$ and a coding potential of approximately 100 open reading frames (ORFs) (10). The family includes five different genera, two of which infect insects while the others infect cold-blooded vertebrates. Species from the genera Megalocytivirus and Lymphocystivirus have been found to infect only teleost fish, while ranaviruses are known to infect reptiles, amphibians, and fish, and the reasons for this broad host specificity are yet unknown.

Ranavirus outbreaks have been described from different locations worldwide. However, full-length genome sequences have been published only for Asian, American, and Australian isolates $(18,21,24,28,35,37,41)$. Recently, it has been proposed that ranaviruses can be subdivided into two distinct groups based on phylogenetic analyses and genome colinearity, grouper iridovirus (GIV)-like ranaviruses and amphibian-like ranaviruses (ALRV) (24). The first group includes GIV and Singapore grouper iridovirus (SGIV), which were isolated in Asia from fish $(35,41)$. The ALRVs include frog virus 3 (FV3) and Ambystoma tigrinum virus (ATV), which were isolated from frogs and salamanders, respectively, in North America, the tiger frog virus (TFV), isolated in China, the epizootic hematopoietic necrosis virus (EHNV), isolated from fish in Australia, and the soft-shelled turtle iridovirus (STIV), isolated in China (21). Within the ALRVs, the degree of genome sequence colinearity is high, although two different groups of viruses can be distinguished: the EHNV/ATV group, which may be closer to a putative most recent common ancestor of the whole group (24), and the FV3/TFV/STIV group, which shows two discrete acquired genomic inversions when compared to the genomes of the former group.

Ranavirus emergence as a pathogen of amphibians and other ectothermic vertebrates is probably linked to their host range plasticity as well as to environmental and ecological factors (17). The first clear evidence that viral infection can be the cause of localized amphibian population declines was reported in Europe, where ranaviruses have caused large-scale mortalities of the common frog Rana temporaria in the United Kingdom since 1985 (40). The causative agent is thought to be a variant of FV3, which may have

\footnotetext{
Received 14 December 2011 Accepted 18 January 2012

Published ahead of print 1 February 2012

Address correspondence to Alí Alejo, alejo@inia.es.

* Present address: Centro de Investigación en Sanidad Animal (INIA), Ctra. de

Algete a El Casar, Valdeolmos, Spain.

C. Mavian and A. López-Bueno contributed equally to this article.

Copyright @ 2012, American Society for Microbiology. All Rights Reserved.

doi:10.1128/JVI.07108-11
} 
been introduced through the movement of infected animals from North America (22).

The common midwife toad virus (CMTV) was first isolated on the European continental mainland in 2007 from diseased tadpoles of the common midwife toad (Alytes obstetricans) in a highaltitude permanent water trough in the Picos de Europa National Park in Spain (4). The virus, causing a high mortality rate in this species as well as in juvenile alpine newts in the 2008 outbreak (Mesotriton alpestris cyreni) (5), was shown to be responsible for a systemic hemorrhagic disease. Common histological findings were the presence of intracytoplasmic inclusion bodies and the necrosis of endothelial cells, the latter of which results in destruction of several organs, including skin, liver, and kidney. Sequence analyses of DNA fragments belonging to the major capsid protein (MCP) and DNA polymerase genes showed that CMTV clustered more closely with the ALRVs than with the GIV-like ranaviruses within the Ranavirus genus. In 2010, a CMTV outbreak in a pond in the Netherlands was described as the cause of a mass mortality event affecting water frogs and common newts (29), showing that both the host range and geographic distribution of CMTV are much wider than previously suspected.

The importance of ranavirus infections in amphibian population declines as well as the lack of knowledge about the nature of circulating European ranaviruses prompted us to determine the complete genome sequence of the emerging CMTV.

\section{MATERIALS AND METHODS}

Virus and cells. CMTV isolated from alpine newts (Mesotriton alpestris cyreni) without plaque purification (4) was amplified in a single step on zebrafish ZF4 cells (ATCC CRL-2050) at $28^{\circ} \mathrm{C}$ in Dutch-modified RPMI medium containing $2 \%$ fetal calf serum. Supernatants from CMTV-infected ZF4 cells were collected, and viral particles were obtained by ultracentrifugation at $20,000 \times g$ through a $36 \%$ sucrose cushion for $1 \mathrm{~h}$ at $4^{\circ} \mathrm{C}$.

Isolation of viral DNA. The virus particles were treated with DNase I and S7 micrococcal nuclease to digest free DNA. After proteinase K treatment, viral DNA was extracted with phenol-chloroform and precipitated with sodium acetate and ethanol in the presence of $10 \mu \mathrm{g}$ of glycogen (Roche) as the carrier. Extracted DNA was randomly amplified using Phi29 DNA polymerase (GenomiPhi V2; GE Healthcare).

Sequencing and assembly of the viral genome. Amplified viral DNA was pyrosequenced on a 454 GS FLX instrument housed in the Parque Científico de Madrid. The output consists of 40,271 sequences with an average size of $375 \mathrm{bp}$. Reads were assembled in two steps with Newbler 2.5.3 (Roche-454 Life Science). First, a de novo assembly under stringent parameters ( $97 \%$ minimum overlap identity in at least $250 \mathrm{bp}$ ) generated 2 contigs flanked by repeated sequences. Then a unique contig was obtained by aligning the reads to the de novo genome under more relaxed parameters ( $98 \%$ identity in at least $50 \mathrm{bp}$ ). PCR amplification and Sanger sequencing were carried out in order to define homopolymer sequencing ambiguities at positions 73,736 and 3,392 and a polymorphism at position 11,812 as well as the precise number of TGTGAAGCGTAAGTCCCC repeats at position 66,494 . The number of repetitions of the microsatellite region detected at position 38,092 was determined by running a specific PCR product in a $4 \%$ agarose gel (see Fig. 2). Sequences of primers are available upon request.

Genome analysis and annotation. Open reading frames (ORFs) were numbered consecutively from the same arbitrary start point as in ATV and $\operatorname{EHNV}(24,28)$, and transcriptional sense was indicated by R (right) or $\mathrm{L}$ (left). The genome was annotated with the genome annotation transfer utility (GATU) software (39) using the genome of STIV as a template and further refined manually by using the similarity search algorithm BLASTP (http://blast.ncbi.nlm.nih.gov/) on all unassigned ORFs longer than $120 \mathrm{bp}$. Overlapping ORFs were annotated only if they had been previously annotated in other ranaviral genomes, preserving the same number for both $\mathrm{R}$ and L orientations (CMTV ORFs 35 and 54). Nucleotide-to-nucleotide comparisons between the CMTV genome and other iridoviral genomes as well as among different ranaviruses were calculated using the JDotter (Java Dot Plot Alignments) software with the default settings. The algorithm employed by this software is described in detail in reference 9 . In the dot plots generated, a straight line represents a stretch of similarity between the two sequences compared on the $x$ and $y$ axes. The full-length genome sequences (accession numbers) used in the dot plot analyses were FV3 (AY548484), TFV (AF389451), ATV (AY150217), EHNV (FJ433873), GIV (AY666015), SGIV (AY521625), and STIV (NC012637). Phylogenetic trees were constructed using MEGA5 software. For the phylogenetic analysis of ranavirus MCPs, the following sequences were used: ranavirus KRV-1 (KRV-1; accession no. ADO14139), Rana catesbeiana virus-JP (RCV-JP; no. BAH80413), softshelled turtle iridovirus (STIV; no. ABC59813), frog virus 3 (FV3; no. ACP19256), tiger frog virus (TFV; no. AAK55105), Bohle iridovirus (BIV; no. ACO90022), pike-perch iridovirus (PPIV; no. ACO90019), Rana esculenta virus (REV; no. ACO90020), Chinese giant salamander virus (CGSV; no. ADZ47908), Ambystoma tigrinum virus (ATV; no. TP003785), epizootic hematopoietic necrosis virus (EHNV; no. AAO32315), Ranavirus maxima/9995205/DNK (Rmax; no. ADI71344), cod iridovirus/15/04.11.92/DNK (CodV; no. ADI71343), European sheatfish virus (ESV; no. ACO90018), European catfish virus (ECV; no. ACO90017), short-finned eel ranavirus (SERV; no. ACO90021), largemouth bass ulcerative syndrome virus (LMBUSV; no. ADB77863), largemouth bass virus (LMBV; no. CBW46836), grouper iridovirus (GIV; no. AEI85923), Singapore grouper iridovirus (SGIV; no. AAS18087), king grouper iridovirus (KGIV; no. AEI85924), crimson snapper iridovirus (CSIV; no. AEI85915), and lymphocystis disease virus from China (LCDV-C; no. YP_025102).

Nucleotide sequence accession number. The genome sequence of CMTV was deposited into GenBank under accession no. JQ231222.

\section{RESULTS}

CMTV is a distinct ranavirus isolated in Europe. To better understand the taxonomic position of the CMTV, we sequenced its MCP gene and performed a phylogenetic analysis comparing the MCP gene to that of 23 other ranaviruses. As shown in Fig. 1A, the CMTV clustered very closely with the Chinese giant salamander virus, isolated in China in 2010 (16), and the Rana esculenta virus, isolated from edible frogs (Pelophylax esculentus) in Italy and Denmark in 2009 (3). Together with the pike-perch iridovirus, isolated from pike-perch fry in Finland in 1998 (38), all four viruses formed a distinct group that was included within the previously described ALRVs but clearly separated from its two proposed major branches, represented by FV3 and EHNV. Collectively, the CMTV-like viruses are found to be more closely related to the FV3 group, suggesting both may have diverged from a common ancestor. Finally, the CMTV was distantly related to other ranaviruses, such as Ranavirus maxima/9995205/DNK, cod iridovirus/15/ 04.11.92/DNK (2), European sheatfish virus (1), and European catfish virus (32), that were isolated from fish on the European continent. As no full-length genome sequences for ranaviruses isolated in Europe have been described to date (Fig. 1B), and given the apparently distinct position of CMTV within the ALRVs, we decided to sequence its complete genome.

CMTV is a typical ALRV. The complete genomic sequence of the CMTV was obtained using pyrosequencing combined with Sanger sequencing of PCR products for unresolved regions. After assembly, we obtained a final genome size of 106,878 bp, with an average coverage of 128 reads per position. The genome size of CMTV is smaller than that of the EHNV (127,011 bp) and slightly 

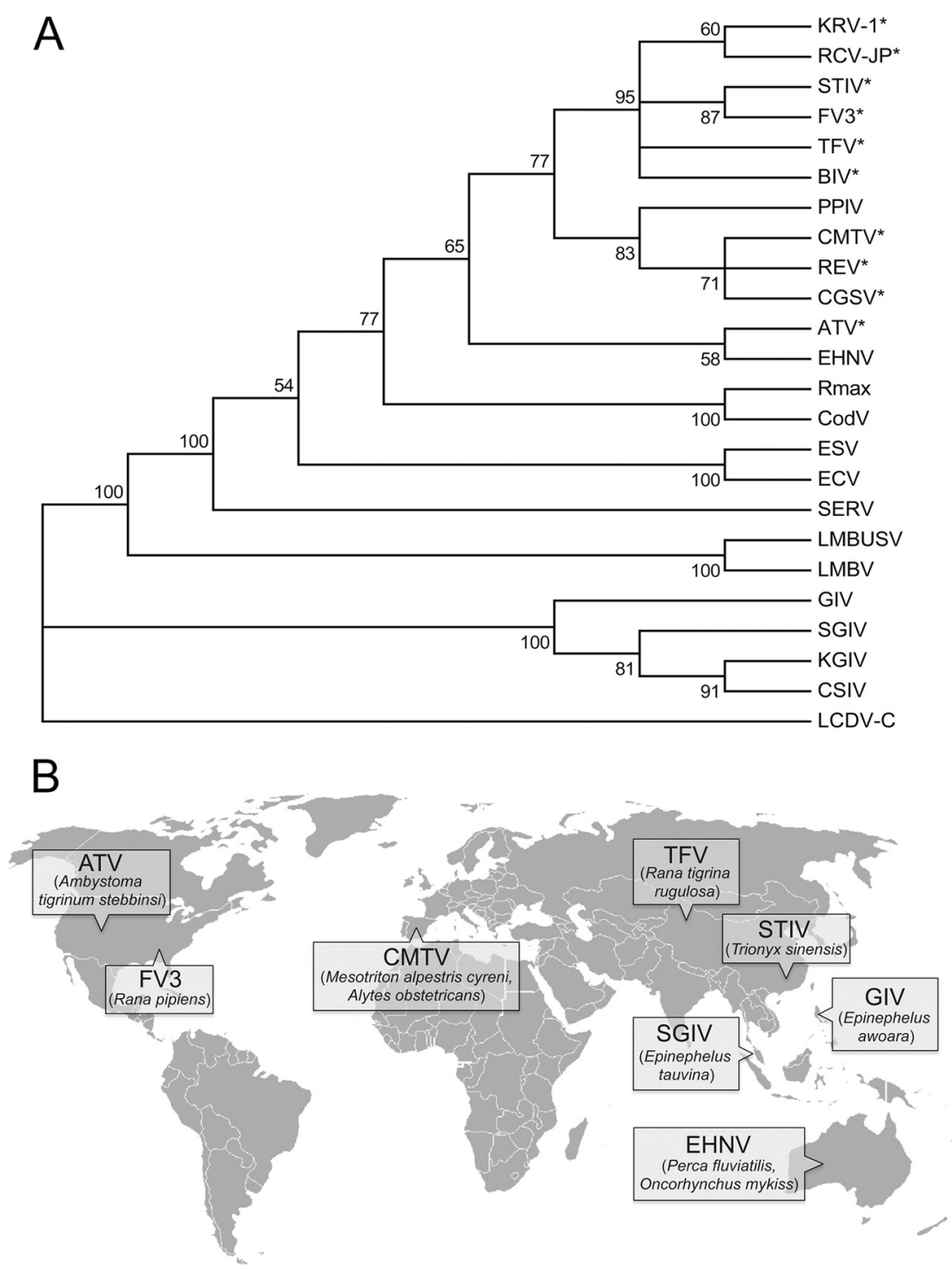

FIG 1 CMTV is a novel amphibian-like ranavirus isolated in Europe. (A) Phylogenetic tree of ranavirus major capsid protein sequences. The sequences were aligned with ClustalW and trimmed manually to the 450 -amino-acid sequence available for the Chinese giant salamander virus (CGSV) MCP, and phylogenetic reconstruction was obtained by neighbor-joining with 1,000 bootstrap replicates. The consensus bootstrap tree is shown, with confidence values indicated on the branches. Branches with bootstrap values below 50\% are collapsed. Lymphocystis disease virus from China (LCDV-C) was used as the outgroup. Viruses infecting amphibian or reptile hosts are marked with an asterisk. The GenBank accession numbers of the Iridovirus MCP sequences used for this analysis are listed in Materials and Methods. (B) World map showing the locations of isolation and the hosts of the published full-length ranavirus genome sequences and CMTV.

larger than those of the other fully sequenced ALRVs ATV $(106,332$ bp), FV3 (105,903 bp), STIV (105,890 bp), and TFV (105,057 bp). The $55.3 \%$ GC content of the CMTV genome is similar to those of the other ALRVs, which range from $54 \%$ to $55 \%$, but higher than those of the GIV (49\%) and SGIV (48\%) ranaviruses.

The microsatellite identified in FV3 (12) and STIV was also found in CMTV. The use of primers specific for the flanking sequences produced a larger PCR amplification product on CMTV DNA than on FV3 DNA, showing that the region in CMTV contains 60 dinucleotide repeats, compared to the 34 copies observed in the other two viruses, and that it can therefore be used for differentiation of these viruses (Fig. 2).

The CMTV genome was found to contain 104 ORFs (Fig. 3) encoding putatively expressed proteins with predicted molecular masses ranging from $5.2 \mathrm{kDa}$ to $144.2 \mathrm{kDa}$ and with conserved

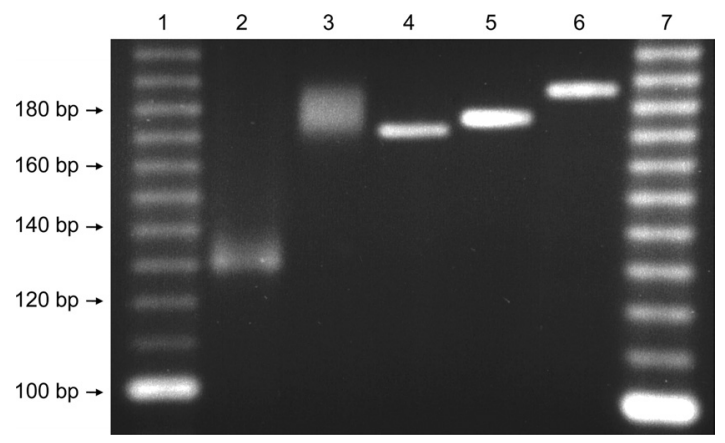

FIG 2 PCR amplification of the microsatellites in CMTV and FV3. The microsatellites of FV3 (lane 2) and CMTV (lane 3) were PCR amplified using specific primers and run on a $4 \%$ agarose gel together with reference PCR products of known sizes (lane 4, $170 \mathrm{bp}$; lane 5, $174 \mathrm{bp}$; lane 6, $184 \mathrm{bp}$ ). Lanes 1 and 7, molecular weight markers. 

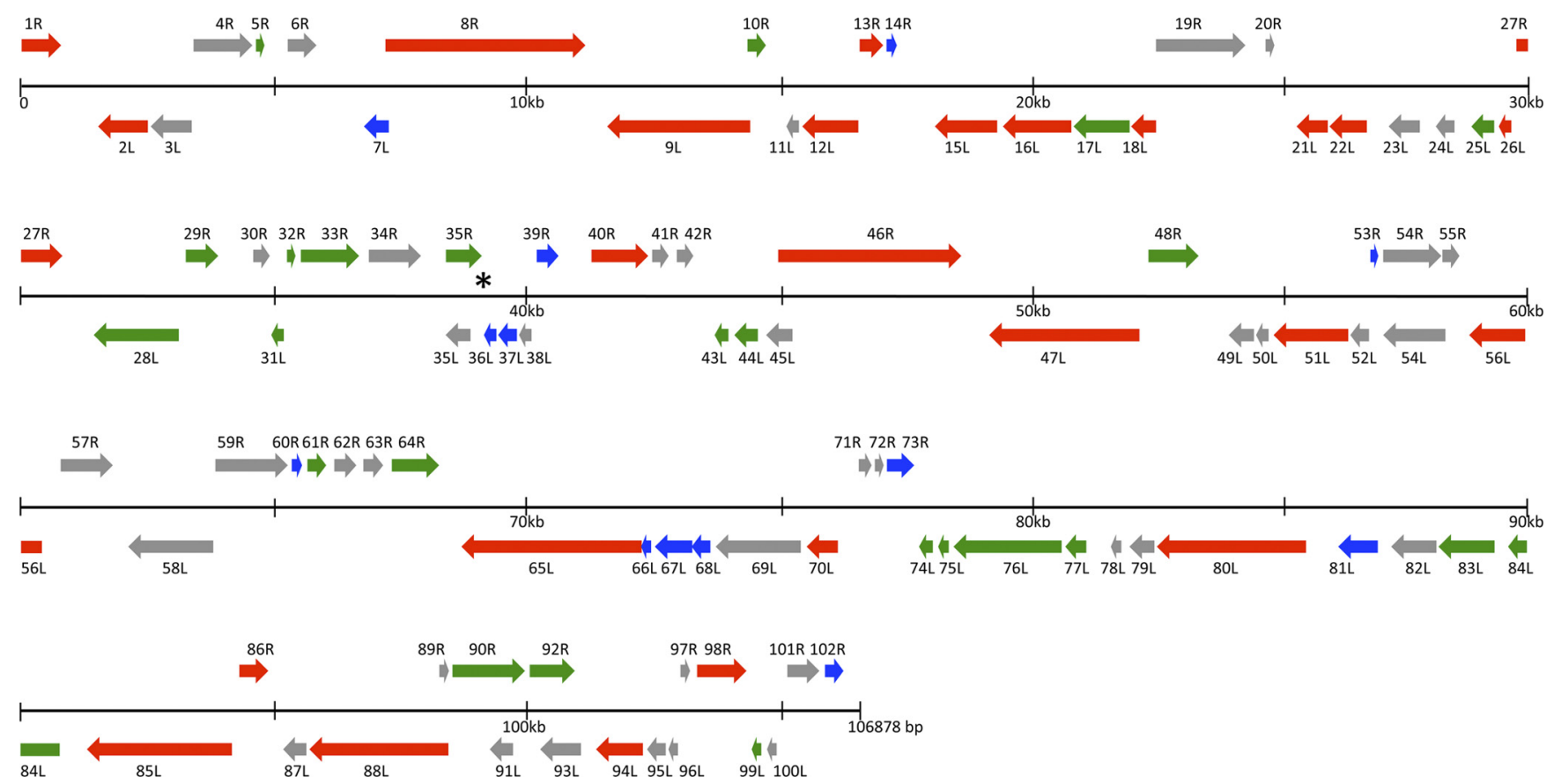

FIG 3 Linear schematic organization of CMTV genome. Predicted open reading frames (ORFs) are represented as arrows indicating the size and direction of transcription. The black lines represent the CMTV genome and are divided into 30-kb segments. ORFs in the forward strand are drawn above the genome line and those in the complement strand are drawn below. Iridovirus core genes, ranavirus-specific genes, and amphibian-like ranavirus-specific genes are indicated in red, green, and blue, respectively. The asterisk shows the position of the microsatellite repetition.

domains assigned to structural proteins as well as proteins potentially involved in replication, transcription, and host response modification (Table 1). For 101 CMTV-predicted proteins, orthologues were readily found in other ranavirus (24) genomes, with identities ranging from $65 \%$ to $100 \%$. Only three ORFs (11L, 96L, and $100 \mathrm{~L}$ ) had not been previously annotated in other ranaviruses, but no significant similarities to other known proteins were found in the databases. Early stop codons or frameshift mutations account for the absence of annotated protein orthologues for these three ORFs in other ALRVs in spite of their relatively high conservation at the nucleotide level. Additionally, a genomic inversion in the FV3 group split the CMTV ORF 100L in these three ranaviruses. Whether these proteins are truly expressed and functional during CMTV infection remains to be addressed.

Analyses of all sequenced iridovirus genomes have identified 26 conserved genes, which make up the core iridovirus genes (15). As expected, these genes are conserved in CMTV. Additionally, 27 more genes were described to be conserved throughout the Ranavirus genus based on the EHNV gene content (24). These include a multigene family with five members in EHNV of which only two are retained in ATV and the FV3 group as well as in CMTV (ORFs $82 \mathrm{~L}$ and $83 \mathrm{~L}$ ). Finally, the set of 13 genes conserved in all ALRVs (24) was also found in CMTV. Altogether, these results show that the CMTV can be classified as a typical ALRV in terms of gene content.

CMTV represents an intermediate in ALRV evolution. Due to the $>90 \%$ identity among ranavirus MCP sequences, phylogenetic studies based exclusively on these sequences may be insufficient for differentiating among ranavirus types. To further establish the phylogenetic relationships between CMTV and other fully sequenced ranaviruses, we performed an analysis of the concate- nated protein sequences derived from the 26 iridoviral core genes (15). As previously reported, the Asian fish viruses GIV and SGIV formed a separated branch within the fully sequenced ranaviruses (Fig. 4). CMTV was found to cluster very confidently within the FV3-like group, which was clearly resolved from the EHNV-like group. Furthermore, CMTV sequences were found to have a slightly higher similarity to the FV3/STIV group than to TFV.

To determine the degree of colinearity of the CMTV genome with those of other ranaviruses, we performed dot plot analyses (Fig. 5 and data not shown). As expected, CMTV did not show major colinearity stretches when compared to the GIV-like fish ranaviruses (data not shown). As described before, ALRVs can be divided into two separate groups in which colinearity is maintained, one including EHNV and ATV and the second including FV3, STIV, and TFV. The CMTV genome was found to not be colinear with either of these groups. Specifically, compared to EHNV, an inversion of the CMTV genomic segment located between positions $\sim 62,000$ and $\sim 91,000$ was observed, while in comparison to $\mathrm{FV} 3$, a single different inversion affecting positions $\sim 15,000$ and $\sim 105,000$ was detected. These sites of genomic rearrangements correspond exactly to those of the double genomic inversion identified previously when FV3 was compared to EHNV (24). This suggests that CMTV or its ancestor may occupy an intermediate position in the evolutionary process that gave rise to the FV3/STIV/TFV group from the EHNV-like ancestor. Thus, inversion of one segment from an EHNV-like precursor may have produced a CMTV ancestor. A further inversion might have then produced the genomic structure found in the FV3/TFV group.

Upon closer inspection of the corresponding dot plots, CMTV was found to contain no major deletions and only three DNA sequence insertions of about 500 bp each compared to FV3 (Fig. 
TABLE 1 Predicted CMTV open reading frames ${ }^{a}$

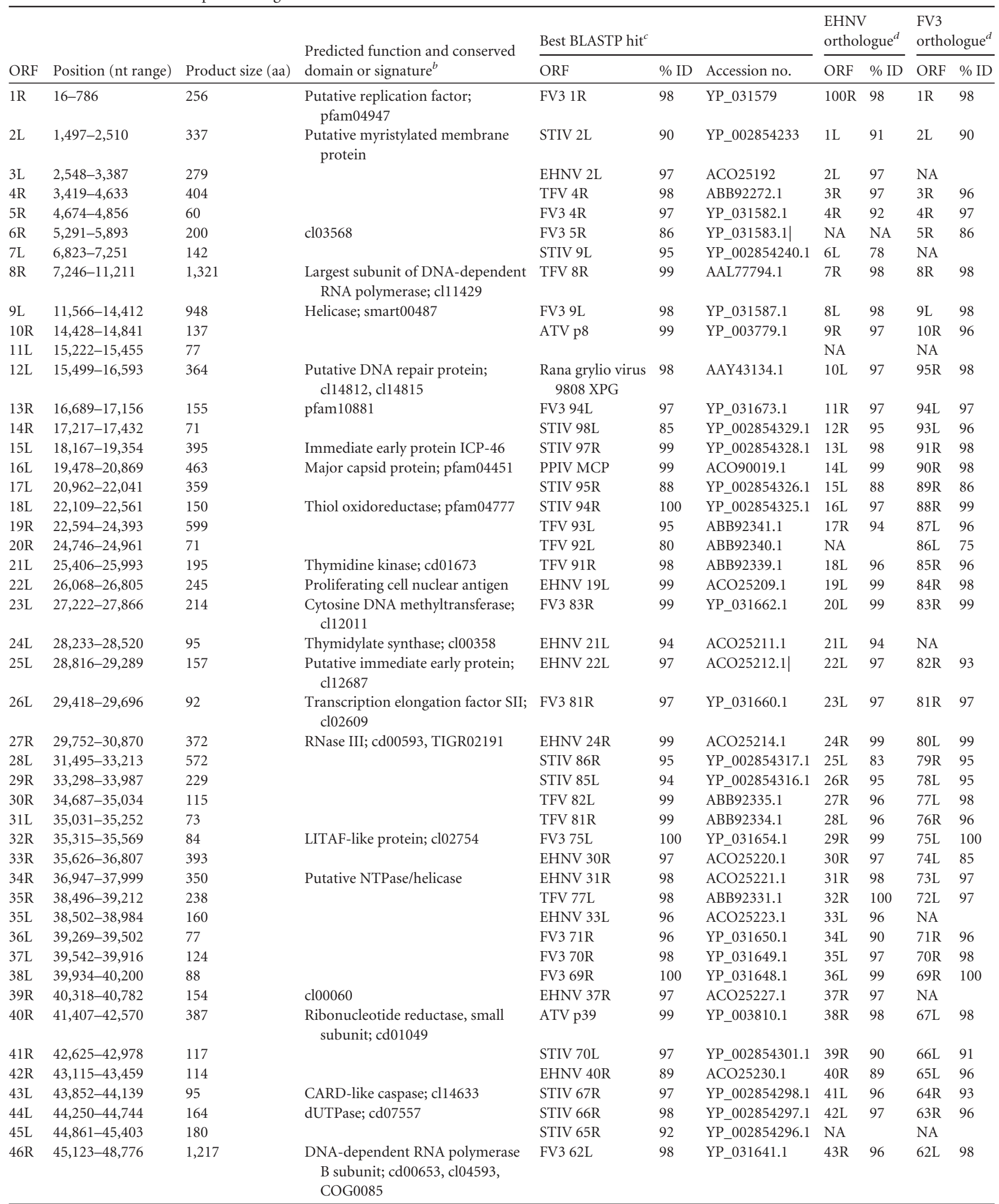


TABLE 1 (Continued)

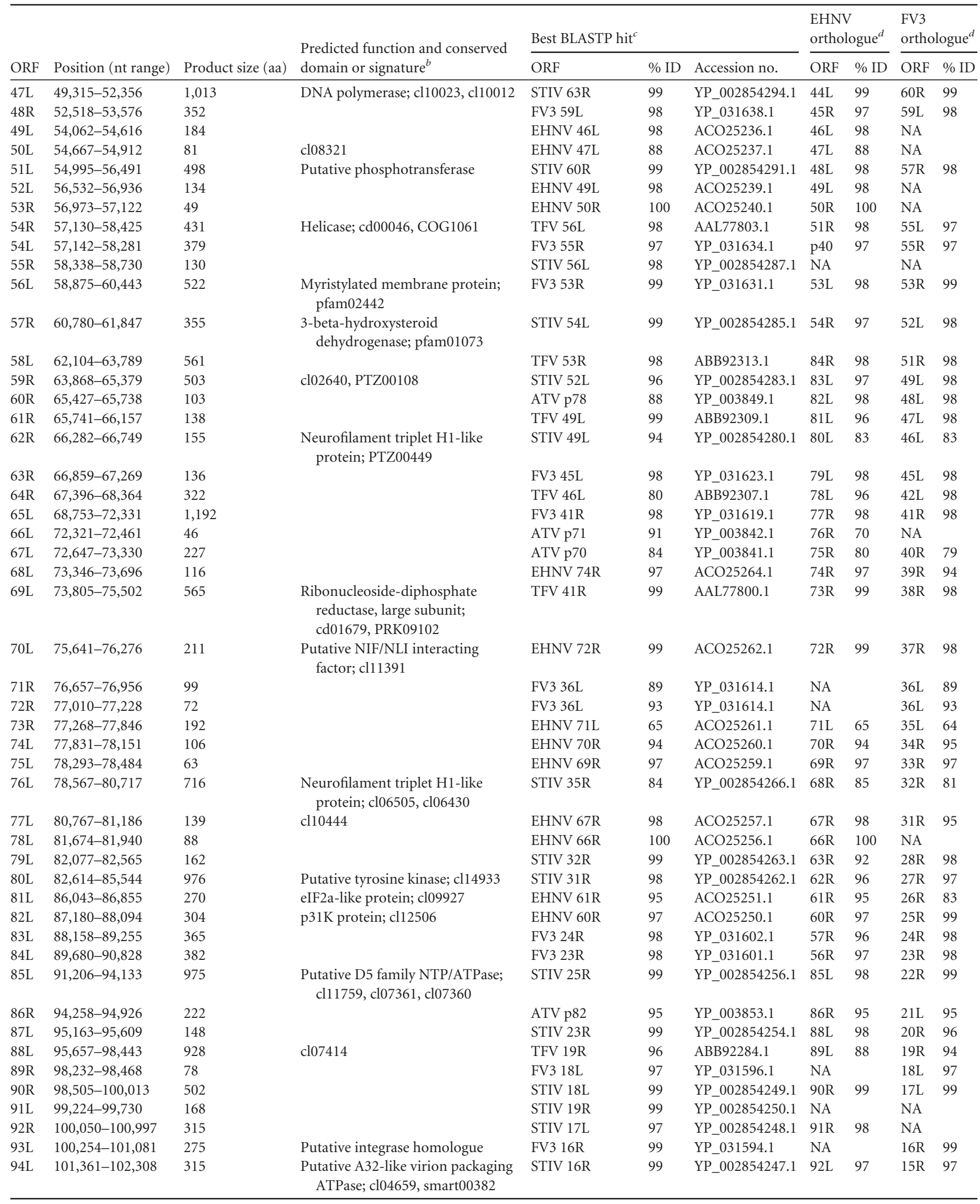




\begin{tabular}{|c|c|c|c|c|c|c|c|c|c|c|}
\hline \multirow[b]{2}{*}{ ORF } & \multirow[b]{2}{*}{ Position (nt range) } & \multirow[b]{2}{*}{ Product size (aa) } & \multirow{2}{*}{$\begin{array}{l}\text { Predicted function and conserved } \\
\text { domain or signature }^{b}\end{array}$} & \multicolumn{3}{|c|}{ Best BLASTP hit ${ }^{c}$} & \multicolumn{2}{|c|}{$\begin{array}{l}\text { EHNV } \\
\text { orthologue }^{d}\end{array}$} & \multicolumn{2}{|c|}{$\begin{array}{l}\text { FV3 } \\
\text { orthologue }^{d}\end{array}$} \\
\hline & & & & ORF & $\%$ ID & Accession no. & ORF & $\%$ ID & ORF & $\%$ ID \\
\hline $96 \mathrm{~L}$ & $102,841-103,035$ & 64 & & & & & NA & & NA & \\
\hline 97R & $103,076-103,270$ & 64 & & TFV 13L & & ABB92279.1 & NA & & NA & \\
\hline $98 \mathrm{R}$ & $103,382-104,425$ & 347 & & STIV 14L & 98 & YP_002854245.1 & $95 \mathrm{R}$ & 97 & $12 \mathrm{~L}$ & 98 \\
\hline $101 \mathrm{R}$ & $105,187-105,873$ & 228 & & EHNV 98R & 97 & ACO25288.1 & $98 \mathrm{R}$ & 97 & $96 \mathrm{R}$ & 94 \\
\hline $102 \mathrm{R}$ & $105,939-106,352$ & 137 & & STIV 103R & 99 & YP_002854334.1 & $99 \mathrm{R}$ & 96 & 97R & 99 \\
\hline
\end{tabular}

${ }^{a}$ nt, nucleotides; aa, amino acids; ID, identity.

${ }^{b}$ Predicted function is based on conserved domains and/or previous annotation in other ranaviruses. LITAF, lipopolysaccharide-induced tumor necrosis factor alpha (TNF- $\alpha$ ).

${ }^{c}$ Significant hits using conserved domain search at NCBI BLASTP are shown.

${ }^{d}$ NA, not applicable (BLASTP E value $>0.001$ ).

5). These insertions corresponded to sequences that are also found in EHNV and to different degrees in ATV and TFV but that are specifically lost in FV3 and STIV. In particular, the insertions affect CMTV 24L, CMTV 39R, and CMTV 81L, which encode a truncated thymidylate synthase protein, a putatively secreted protein containing an intact FGF domain, and an eIF2 $\alpha$-like protein, respectively. Conversely, compared to EHNV, CMTV presents at least 14 deletions and two insertions, which affect CMTV 6R as well as CMTV 71R and CMTV 72R, that are also found in TFV, FV3, and STIV. This pattern of sequence gain and loss among ALRVs is also consistent with the intermediate position of CMTV in the evolution of EHNV-like viruses toward FV3-like viruses, since CMTV retains EHNV-like characteristics which are lost in FV3-like viruses and has already acquired sequences found previously only in FV3-like viruses. Therefore, the genomic content and structure of CMTV may resemble those of the last common ancestor of TFV, STIV, and FV3 viruses.

\section{DISCUSSION}

In this report, we have shown that the CMTV, the first European ranavirus to be completely sequenced, should be classified as an ALRV. Based on our analyses, we propose that the CMTV might

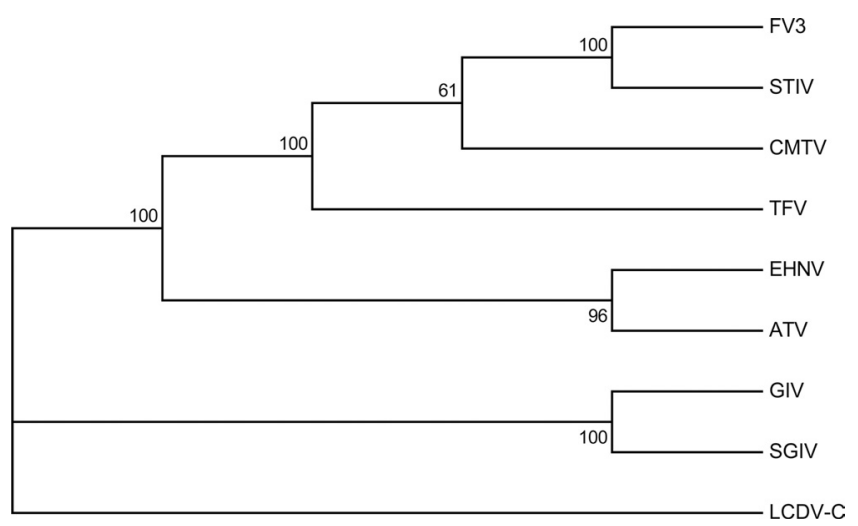

FIG 4 CMTV is closely related to the FV3-like viruses. Phylogenetic analysis of the concatenated sequences of the 26 iridovirus core proteins (15) from the indicated viruses. The sequences were aligned with ClustalW, and the phylogenetic reconstruction was obtained by neighbor-joining with 1,000 bootstrap replicates. Consensus bootstrap confidence values are indicated above the branches. LCDV-C was used as the outgroup. represent, in terms of gene content and genomic structure, a very recent ancestor of the previously described group of FV3-like viruses (24). As the CMTV forms a cluster with ranaviruses isolated from different species and locations worldwide, including fish and amphibian viruses, we believe that these may form a single, novel group of ALRVs. The information provided by this report should therefore be useful to specifically address the relationship of these viruses with CMTV and to further define the taxonomic positions of different ranavirus isolates, which have been poorly described at the genetic level.

One of the most outstanding features of ranaviruses is their wide host range, which includes anuran and urodele amphibians, reptiles, and teleost fish. It has been proposed that the most recent common ancestor of all ALRVs was most probably a fish virus, with independent events giving rise to isolates infecting different hosts (24). Experimental evidence suggests that ATV may infect only urodele hosts, while neither fish nor frogs are susceptible to ATV infection (25). As CMTV can infect both anuran and urodele amphibians in the wild $(4,5,29)$, it is tempting to speculate that CMTV may have derived from an ATV-related virus, acquiring the ability to infect frogs. Potentially, further divergence into the FV3-like viruses may have produced frog- and reptile-specific viruses. However, FV3 infections of both fish and salamander species have been reported (30), and the Bohle iridovirus, isolated from the ornate burrowing frog in Australia, is also known to infect fish (31). Therefore, both data from experimental infections and a clearer taxonomic description of ranaviruses based on complete genome information will help address this issue.

As described above, CMTV has retained some features from the EHNV-like group which are lost in FV3-like viruses and has acquired potential coding sequences which were previously identified only in the FV3-like viruses, further supporting their intermediate position in the evolution between these groups of ALRVs. Interestingly, some of these coding regions may play a role in host range or virulence. In particular, a potentially functional protein belonging to the fibroblast growth factor family is present only in EHNV, ATV, and CMTV. In baculoviruses, such an activity was shown to be able to induce cell migration, suggesting a role in immunomodulation or viral spread (13). Additionally, the eIF $2 \alpha$-like protein (vIF $2 \alpha \mathrm{H})$ present in most ALRVs, including CMTV, but truncated in the FV3 and STIV has been demonstrated to act as an inhibitor of the antiviral protein kinase PKR (33). Thus, ATV mutants lacking 


\section{CMTV}

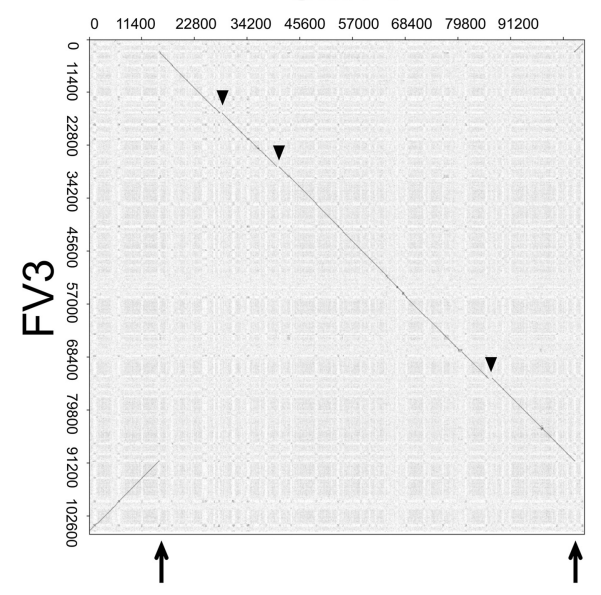

CMTV

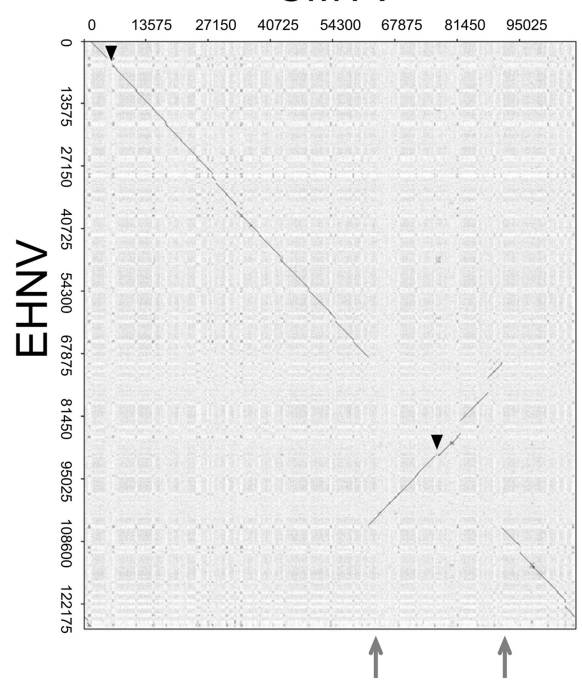

FV3

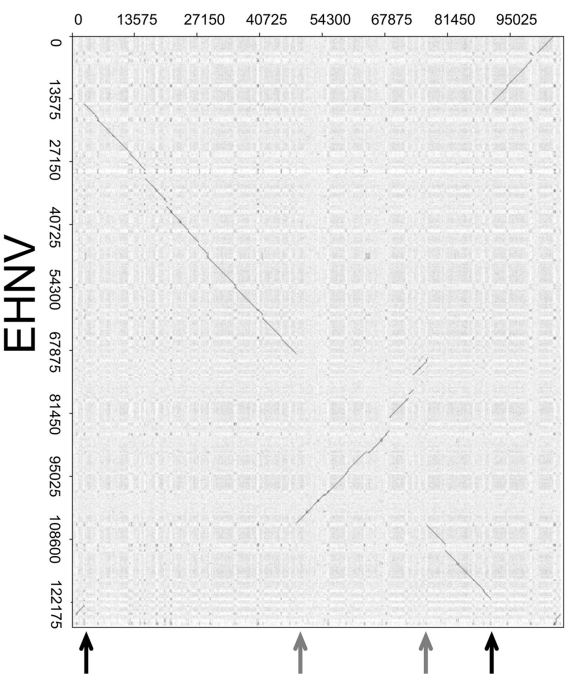

FIG 5 CMTV is an evolutionary intermediate in ALRVs. Dot plot comparisons of the CMTV genome and the EHNV and FV3 genomes. For clarity, the reverse complement sequence of the FV3 genome was used in these comparisons. As a reference, a dot plot of EHNV versus FV3 is shown in the right panel. Black arrows indicate the sites of inversion in the CMTV genome compared to the FV3 genome, and gray arrows indicate the sites of inversion in the CMTV genome compared to the EHNV genome. Triangles indicate sequences present in CMTV but absent in FV3 or EHNV.

vIF $2 \alpha \mathrm{H}$ activity were shown to be more sensitive to the antiviral activity of interferon (IFN) and displayed an increased time to death in infected salamanders, demonstrating the protein's role as an important virulence factor in vivo (27). Conversely, CMTV 6R, which is found in ALRVs except EHNV and ATV, shows significant similarity to DNA and protein sequences from frog species, suggesting a recent acquisition event during the process of adaptation to novel amphibian hosts. Implications of these specific events of sequence gain and loss among ALRV groups on virus host range or pathogenicity remain to be addressed.

Whether the broad host range of ranaviruses, compared to that of other double-stranded DNA viruses like orthopoxviruses, is an inherent feature of the iridoviruses or related to a more recent evolutionary history of this virus family is an important topic for study. While gene loss has been established as one of the main driving forces in poxvirus evolution (19), we observe very little differences in terms of global gene content among ALRVs, which might indicate that this group of viruses is only on the verge of an evolutionary radiation. This may relate to the human-mediated exchange of host species or viruses between distant regions, which increases the number of potential hosts to infect and is consistent with their established character as emerging infectious-disease-causing agents.

As pathogens of wildlife, ranaviruses have been found to cause mass mortalities mainly in amphibian populations worldwide. One of the earliest amphibian population declines to be reported was that affecting the common midwife toad and other amphibian species, including salamanders, during the last decade in Spain $(7,8)$. These declines have been associated with the emergence of chytridiomycosis, a disease caused by the fungal pathogen Batrachochytrium dendrobatidis. Recent studies have shown the widespread yet heterogeneous distribution of Batrachochytrium dendrobatidis across the Iberian Peninsula and predict further spread of the disease in the future (42). However, the isolation of CMTV from diseased amphibians in Spain suggests that ranavirus infection, too, may be having an impact on population declines, as reported for other locations. In the Nether- lands, animals affected with CMTV showed no evidence of Batrachochytrium dendrobatidis infection (29), suggesting that the two pathogens did not occur simultaneously. Whether an interaction between the pathogens is important for amphibian extinction dynamics remains to be addressed. Mass mortality events affecting the urodele Triturus marmoratus in northwest Portugal in 2003 are probably also related to ranavirus outbreaks, and recently, a novel ranavirus closely related to FV3 was isolated from lizards in Portugal (12). The nature of these viruses and whether they may represent different isolates of the CMTV are currently unknown. Although the distribution and degree of prevalence of CMTV in different host species need to be studied in greater detail, the presence of CMTV in the Netherlands (29) suggests that this virus may show a wide geographic distribution across the European mainland. In the case of tiger salamander infections in North America, it was shown that while ATV has coevolved with its host in their geographic range, human-mediated transfer of ATV-infected bait salamanders introduced novel, more virulent strains to discrete locations, resulting in disease emergence $(26,36)$. It will be important to determine the mechanisms of emergence of CMTV-caused disease and whether it may be linked to environmental factors, virus spread, or both.

As advances in sequencing technologies make more genomic information on the ranavirus isolates available, a better understanding of the evolutionary history of this virus family will be gained. In particular, a better knowledge of the ranavirus species and strain structure will be crucial to set up detection methods and surveillance strategies in order to minimize their impact on amphibian wildlife and cultivated species.

\section{ACKNOWLEDGMENTS}

This work was supported by grant AGL 2009-08711 from the Spanish Ministerio de Ciencia e Innovación. Alberto López-Bueno and Carla Mavián are recipients of the Ramón y Cajal and Formación del Personal Investigador fellowships, respectively, from the same institution. 


\section{REFERENCES}

1. Ahne W, Schlotfeldt HJ, Thomsen I. 1989. Fish viruses: isolation of an icosahedral cytoplasmic deoxyribovirus from sheatfish (Silurus glanis). Zentralbl. Veterinarmed. B 36:333-336.

2. Ariel E, Holopainen R, Olesen NJ, Tapiovaara H. 2010. Comparative study of ranavirus isolates from cod (Gadus morhua) and turbot (Psetta maxima) with reference to other ranaviruses. Arch. Virol. 155:1261-1271.

3. Ariel E, et al. 2009. Ranavirus in wild edible frogs Pelophylax kl. esculentus in Denmark. Dis. Aquat. Organ. 85:7-14.

4. Balseiro A, et al. 2009. Pathology, isolation and molecular characterisation of a ranavirus from the common midwife toad Alytes obstetricans on the Iberian Peninsula. Dis. Aquat. Organ. 84:95-104.

5. Balseiro A, et al. 2010. Outbreak of common midwife toad virus in alpine newts (Mesotriton alpestris cyreni) and common midwife toads (Alytes obstetricans) in northern Spain: a comparative pathological study of an emerging ranavirus. Vet. J. 186:256-258.

6. Berger L, et al. 1998. Chytridiomycosis causes amphibian mortality associated with population declines in the rain forests of Australia and Central America. Proc. Natl. Acad. Sci. U. S. A. 95:9031-9036.

7. Bosch J, Martínez-Solano I. 2006. Chytrid fungus infection related to unusual mortalities of Salamandra salamandra and Bufo bufo in the Peñalara Natural Park, Spain. Oryx 40:84-89.

8. Bosch J, Martínez-Solano I, García-París M. 2001. Evidence of a chytrid fungus infection involved in the decline of the common midwife toad (Alytes obstetricans) in protected areas of central Spain. Biol. Conserv. 97:331-337.

9. Brodie R, Roper RL, Upton C. 2004. JDotter: a Java interface to multiple dotplots generated by dotter. Bioinformatics 20:279-281.

10. Chinchar VG, Hyatt A, Miyazaki T, Williams T. 2009. Family Iridoviridae: poor viral relations no longer. Curr. Top. Microbiol. Immunol. 328: 123-170.

11. Collins JP. 2010. Amphibian decline and extinction: what we know and what we need to learn. Dis. Aquat. Organ. 92:93-99.

12. de Matos AP, et al. 2011. New viruses from Lacerta monticola (Serra da Estrela, Portugal): further evidence for a new group of nucleo-cytoplasmic large deoxyriboviruses. Microsc. Microanal. 17:101-108.

13. Detvisitsakun C, Berretta MF, Lehiy C, Passarelli AL. 2005. Stimulation of cell motility by a viral fibroblast growth factor homolog: proposal for a role in viral pathogenesis. Virology 336:308 -317.

14. Duffus AL. 2009. Chytrid blinders: what other disease risks to amphibians are we missing? Ecohealth 6:335-339.

15. Eaton HE, et al. 2007. Comparative genomic analysis of the family Iridoviridae: re-annotating and defining the core set of iridovirus genes. Virol. J. 4:11.

16. Geng Y, et al. 2011. First report of a ranavirus associated with morbidity and mortality in farmed Chinese giant salamanders (Andrias davidianus). J. Comp. Pathol. 145:95-102.

17. Gray MJ, Miller DL, Hoverman JT. 2009. Ecology and pathology of amphibian ranaviruses. Dis. Aquat. Organ. 87:243-266.

18. He JG, et al. 2002. Sequence analysis of the complete genome of an iridovirus isolated from the tiger frog. Virology 292:185-197.

19. Hendrickson RC, Wang C, Hatcher EL, Lefkowitz EJ. 2010. Orthopoxvirus genome evolution: the role of gene loss. Viruses 2:1933-1967.

20. Hof C, Araujo MB, Jetz W, Rahbek C. 2011. Additive threats from pathogens, climate and land-use change for global amphibian diversity. Nature 480:516-519.

21. Huang Y, et al. 2009. Complete sequence determination of a novel reptile iridovirus isolated from soft-shelled turtle and evolutionary analysis of Iridoviridae. BMC Genomics 10:224

22. Hyatt AD, et al. 2000. Comparative studies of piscine and amphibian iridoviruses. Arch. Virol. 145:301-331.

23. IUCN. 2011. Numbers of threatened species by major groups of organisms (1996-2011), version 2011.2. http://www.iucnredlist.org/documents /summarystatistics/2011_2_RL_Stats_Table1.pdf.

24. Jancovich JK, Bremont M, Touchman JW, Jacobs BL. 2010. Evidence for multiple recent host species shifts among the ranaviruses (family Iridoviridae). J. Virol. 84:2636-2647.

25. Jancovich JK, Davids EW, Seiler A, Jacobs BL, Collins JP. 2001. Transmission of the Ambystoma tigrinum virus to alternative hosts. Dis. Aquat. Organ. 46:159-163.

26. Jancovich JK, et al. 2005. Evidence for emergence of an amphibian iridoviral disease because of human-enhanced spread. Mol. Ecol. 14:213-224.

27. Jancovich JK, Jacobs BL. 2011. Innate immune evasion mediated by the Ambystoma tigrinum virus eukaryotic translation initiation factor $2 \alpha$ homologue. J. Virol. 85:5061-5069.

28. Jancovich JK, et al. 2003. Genomic sequence of a ranavirus (family Iridoviridae) associated with salamander mortalities in North America. Virology 316:90-103.

29. Kik M, et al. 2011. Ranavirus-associated mass mortality in wild amphibians, the Netherlands, 2010: a first report. Vet. J. 190:284-286.

30. Mao J, Green DE, Fellers G, Chinchar VG. 1999. Molecular characterization of iridoviruses isolated from sympatric amphibians and fish. Virus Res. 63:45-52.

31. Moody NJG, Owens L. 1994. Experimental demonstration of the pathogenicity of a frog virus, Bohle iridovirus, for a fish species, barramundiLates-calcarifer. Dis. Aquat. Organ. 18:95-102.

32. Pozet F, Morand M, Moussa A, Torhy C, de Kinkelin P. 1992. Isolation and preliminary characterization of a pathogenic icosahedral deoxyribovirus from the catfish Ictalurus melas. Dis. Aquat. Organ. 14:35-42.

33. Rothenburg S, Chinchar VG, Dever TE. 2011. Characterization of a ranavirus inhibitor of the antiviral protein kinase PKR. BMC Microbiol. 11:56.

34. Sodhi NS, et al. 2008. Measuring the meltdown: drivers of global amphibian extinction and decline. PLoS One 3:e1636.

35. Song WJ, et al. 2004. Functional genomics analysis of Singapore grouper iridovirus: complete sequence determination and proteomic analysis. J. Virol. 78:12576-12590.

36. Storfer A, et al. 2007. Phylogenetic concordance analysis shows an emerging pathogen is novel and endemic. Ecol. Lett. 10:1075-1083.

37. Tan WG, Barkman TJ, Gregory Chinchar V, Essani K. 2004. Comparative genomic analyses of frog virus 3 , type species of the genus Ranavirus (family Iridoviridae). Virology 323:70-84.

38. Tapiovaara H, Olesen NJ, Linden J, Rimaila-Parnanen E, von Bonsdorff CH. 1998. Isolation of an iridovirus from pike-perch Stizostedion lucioperca. Dis. Aquat. Organ. 32:185-193.

39. Tcherepanov V, Ehlers A, Upton C. 2006. Genome annotation transfer utility (GATU): rapid annotation of viral genomes using a closely related reference genome. BMC Genomics 7:150.

40. Teacher AGF, Cunningham AA, Garner TWJ. 2010. Assessing the longterm impact of Ranavirus infection in wild common frog populations. Anim. Conserv. 13:514-522.

41. Tsai CT, et al. 2005. Complete genome sequence of the grouper iridovirus and comparison of genomic organization with those of other iridoviruses. J. Virol. 79:2010-2023.

42. Walker SF, et al. 2010. Factors driving pathogenicity vs. prevalence of amphibian panzootic chytridiomycosis in Iberia. Ecol. Lett. 13:372-382. 